\title{
Treating refractory leukemias in childhood, role of clofarabine
}

\author{
Theresa M Harned \\ Paul S Gaynon
}

Department of Hematology-Oncology, Childrens Hospital Los Angeles, Los Angeles, CA, USA
Correspondence:Theresa M Harned 4650 Sunset Blvd MS 54, Los Angeles, CA 90027, USA

Tel + $32336|2| 2 \mid$

Fax +13233617128

Email tharned@chla.usc.edu

\begin{abstract}
Approximately 4000 children and adolescents under the age of 20 years develop acute leukemia per year in the US. Acute lymphoblastic leukemia (ALL) is the most common pediatric cancer. Despite impressive improvements in outcome, relapsed ALL is the fourth most common pediatric malignancy. Therapy for relapsed ALL remains unsatisfactory, and the majority of relapse patients still succumb to leukemia. Between one-third and one-half of patients with acute myelogenous leukemia (AML) relapse, and no standard therapy is recognized for patients with relapsed and/or refractory AML. Novel therapeutic agents are needed to improve the cure rate for relapsed ALL and AML. Clofarabine is a next-generation nucleoside analog, designed to incorporate the best features and improve the therapeutic index of cladribine and fludarabine. Clofarabine inhibits both DNA polymerase and ribonucleotide reductase, leading to impaired DNA synthesis and repair, and directly induces apoptosis. Phase I and II single-agent trials in children have shown that clofarabine is safe and active in both myeloid and lymphoid relapsed/refractory acute leukemias. Clofarabine has been approved by the FDA for pediatric patients with relapsed/refractory ALL after at least 2 prior therapeutic attempts. Rational combinations of clofarabine with other active agents in refractory leukemias are currently under investigation.
\end{abstract}

Keywords: clofarabine, leukemia, refractory, pediatric, childhood

\section{Introduction}

Approximately 4000 children and adolescents under the age of 20 years develop acute leukemia per year in the US. Acute lymphoblastic leukemia (ALL) is the most common pediatric cancer, accounting for one-fourth of all childhood cancers and approximately $75 \%$ of all cases of childhood leukemia. In the US alone, approximately 3000 cases of pediatric ALL are diagnosed annually, with an incidence of 29.2 cases per million per year (Grovas et al 1997; Jemal et al 2004).

Primary treatment of pediatric ALL is "risk-adapted," ie, treatment allocation is based on an estimate of risk of relapse based on presenting features, eg, age at diagnosis, initial white blood cell count, immunophenotype, and cytogenetics and response to therapy, eg, disappearance of peripheral blasts, microscopic marrow response, and/or laboratory measurement submicroscopic leukemia, termed minimal residual disease (MRD) (Schultz et al 2007). Increasingly effective post induction intensification employing conventional agents has steadily improved event-free survival. According to the US population-based Surveillance and End Results survey, 5-year survival for children aged 1-14 years has increased from 53\% in 1974-76 to 85\% in 1992-1999 (Jemal et al 2004).

Despite the impressive improvements in outcome, relapsed ALL remains the fourth most common pediatric malignancy, with an incidence of approximately 6 cases per million in 2007 (Gaynon et al 1998). Therapy for patients with relapsed ALL remains unsatisfactory, especially for patients with early bone marrow relapse 
within 3 years of diagnosis (Gaynon et al 1998; Nguyen et al 2006). Second remissions are common. Four-drug therapy with vincristine, prednisone, asparaginase, and doxorubicin induces complete remissions in $38 \%, 80 \%$, and $95 \%$ of children with initial remissions $<18$ months, $18-36$ months, and $>36$ months, respectively. Of patients achieving CR2, $80 \%$ remain MRD positive at $10^{-4}$ by flow cytometry after CR $1<36$ months compared to $50 \%$ positive for $\mathrm{CR} 1>36$ months (Raetz et al 2006). Therapeutic options after second remission (CR2) include further chemotherapy and/or hematopoietic stem cell transplantation (HSCT) (Eapen et al 2006; Gaynon et al 2006).

However, the majority of relapse patients still succumb to leukemia. For patients enrolled on Children's Cancer Group (CCG) 1900 series studies between 1997 and 2002, the 3-year survival after marrow, CNS, and testes relapse was $28 \%, 60 \%$, and 60\%, respectively (Nguyen et al 2006). Adverse prognostic factors after relapse include early versus late timing, marrow versus extramedullary relapse, and T-cell versus B-precursor immunophenotype (Chessells 1998; Eckert et al 2001; Chessells et al 2003; Einsiedel et al 2005). MRD after re-induction chemotherapy, as measured by PCR or flow cytometry, may be predictive of outcome in CR2 after marrow relapse (Eckert et al 2001; Coustan-Smith et al 2004). Higher levels of pre-transplant MRD may predict relapse after hematopoietic stem cell transplant (HSCT) (Knechtli et al 1998; Bader et al 2002; Goulden et al 2003). Better pre-transplant chemotherapy is needed that allows more patients to proceed to transplant with lower MRD and less deep seated infection or organ damage.

Although third remissions are attained with some frequency, outcomes for patients with second or subsequent relapse remain poor (Chessells 1998; Chessells et al 2003). Several drug combinations have significant CR rates in refractory first relapse, or second or subsequent relapse (Table 1). These CR rates cluster around $40 \%$, despite the

Table I Complete response rates in second and subsequent relapse $A L L$

\begin{tabular}{|c|c|c|}
\hline Regimen & CR rate (\%) & Reference \\
\hline POG 8866 vincristine, & $30 / 74(4 I)$ & Kurtzberg et all 993 \\
\hline Prednisone, L-asparaginase & & \\
\hline POG 9160 idarubicin,cytarabine & $30 / 82(37)$ & Bernstein et al 1997 \\
\hline $\begin{array}{l}\text { High-dose cytarabine, } \\
\text { L-asparaginase }\end{array}$ & $22 / 52(42)$ & Harris et al 1998 \\
\hline Ifosfamide, etoposide & $8 / 20(40)$ & Crooks and Sato 1995 \\
\hline $\begin{array}{l}\text { Topotecan, vinorelbine, thiotepa, } \\
\text { Dexamethasone and gemcitabine }\end{array}$ & $6 / 17(35)$ & $\begin{array}{l}\text { Kolb and Steinherz } \\
2003\end{array}$ \\
\hline
\end{tabular}

Derived from Gaynon 2005.

Abbreviations: ALL, acute lymphoblastic leukemia. use of a variety of drugs with different putative mechanisms of action (Gaynon 2005). An overall 8\% survival rate was reported among patients who achieved third remission after a second marrow relapse (Chessells et al 2003).

We have proposed that candidate drug combinations with CR rates $\leq 40 \%$ are unlikely to be useful for improving cure rates. Alternatively, novel regimens with $\mathrm{CR}$ rates $>60 \%$ would be of compelling interest (Gaynon 2005). Although data are lacking for CR3 and subsequent remissions, measurement of minimal residual disease (MRD) may be useful to assess response.

Acute myelogenous leukemia (AML) comprises 15\%-20\% of childhood leukemia, but accounts for more than $30 \%$ of deaths from leukemia. In the US, approximately 1000 children per year are diagnosed with AML, with an incidence of between 5 and 7 cases per million per year (Grovas et al 1997; Jemal et al 2004). Better supportive care and better use of conventional agents have improved outcomes in childhood AML. Current therapy for patients with newly diagnosed AML involves induction therapy with myelosuppressive cytotoxic agents, including cytarabine and an anthracycline or anthracinedione, and post-remission intensification with chemotherapy or HSCT. Three recent large multi-center pediatric studies yielded remission induction rates of approximately $90 \%$. Five-year event-free survival is nearing or surpassing $50 \%$ (Aladjidi et al 2003; Creutzig et al 2005, 2006; Gibson et al 2005). Risk-group stratification based on cytogenetic features, FLT-3 expression (Pollard et al 2006), and response to therapy is becoming increasingly important.

One-third to one-half of patients with AML relapse. Most relapses are isolated to the bone marrow, and are more likely to occur in the first year after diagnosis. No standard therapy is recognized for patients with relapsed and/or refractory AML. Regimens that contain high-dose cytarabine, often in combination with other agents such as mitoxantrone, etoposide, fludarabine, or 2-chlorodeoxyadenosine, have significant activity (Golub et al 2006). Gemtuzamab ozogamicin, a monoclonal antibody directed against the CD33 differentiation antigen and coupled to genotoxic calicheamicin, has shown activity in relapsed and refractory AML in both children and adults (Sievers et al 2001; Zwaan et al 2003; Reinhardt et al 2004; Arceci et al 2005). Gemtuzamab is currently being studied in newly diagnosed children and adults in combination with multiagent chemotherapy (Burnett et al 2006).

Perhaps $50 \%$ of patients with primary induction failure will achieve CR with subsequent therapy (Wells et al 2003) and $70 \%$ of patients with CR1 achieve a second remission employing similar drugs to those used initially (Webb 1999; 
Aladjidi et al 2003; Wells et al 2003). However, long-term survival for children with relapsed AML ranges between 20\% and 33\% (Aladjidi et al 2003; Castellino et al 2007). Prognosis is related to specific cytogenetic abnormalities and the duration of first remission. Patients relapsing after a CR1 $>1$ year have a better survival. The need remains for therapeutic options which are both less toxic and more effective than the conventional treatment.

\section{New agents for relapsed and refractory childhood leukemias}

Novel therapeutic agents are needed to improve the cure rate of patients with relapsed or refractory ALL and AML. Emerging new treatments for ALL include next generation nucleoside analogs such as the deoxyadenosine analog clofarabine (Clolar ${ }^{\circledR}$, Genzyme), which was approved by the FDA in December 2004 for the treatment of pediatric patients with relapsed or refractory ALL who have received at least two prior chemotherapy regimens (Pui and Jeha 2005), and the deoxyguanosine analog nelarabine (Arranon ${ }^{\circledR}$, GlaxoSmithKline), which has been approved by the FDA for third-line treatment of patients with T-cell leukemia or lymphoma (Kurtzberg 2007). In acute promyelocytic leukemic, arsenic trioxide has shown benefit in randomized trials (Powell et al 2006, 2007). In AML, preliminary data suggest an EFS advantage for addition of gemtuzumab to conventional induction chemotherapy (Burnett et al 2006) and an induction rate advantage for addition of the FLT-3 inhibitor lestaurtinib for patients with AML with FLT-3 internal tandem repeats (Levis et al 2005) . Other promising new agents include monoclonal antibodies against leukemiaspecific antigens, such as rituximab, a humanized anti-CD20 antibody, and epratuzamab, targeting CD22.

Over 100 gene mutations and genetic abnormalities have been reported in AML, reflecting the heterogeneity of the disease and the presence of multiple active pathways (Tallman 2006). Mutations that activate signal transduction pathways lead to proliferative survival advantages and may be targeted with small molecule inhibitors of the specific mutations, such as FLT3, C-Kit, and RAS, while mutations that lead to inhibition of differentiation may be targeted with differentiating agents (eg, all-trans-retinoic acid [ATRA] for patients with acute promyelocytic leukemia [APL]). Targeted therapy may interfere with host support for the leukemia cell. The mechanisms of treatment failure in ALL remain to be elucidated. One does well to remember that neither ATRA alone nor imatinib alone is curative for APL or chronic myelogenous leukemia, respectively. A gene may be over-expressed, yet not critically necessary for leukemic de-differentiation or proliferation.

New agents have been developed for multiple molecular targets in AML, including antibodies to cell surface antigen CD33, inhibitors of multi-drug resistance proteins, farnesyl transferase inhibitors, FLT3-targeted tyrosine kinase inhibitors, and inhibitors of the antiapoptotic protein Bcl-2 and the mammalian target of rapamycin (mTOR). New alkylating agents and purine analogs such as cloretazine and clofarabine, which target DNA and ribonucleotide reductases, respectively, are also under investigation. Targeted agents will likely need to be used in combination with other targeted agents and/or cytotoxic agents to inhibit the multiple pathways present in AML and to improve response rates (Tallman 2006). Clinical benefit will require the right stuff, ie, the right drug be used against the right target in the right disease at the right dose and schedule in the right population with the right accompanying therapy (Gaynon 2005). Clinical development of these therapies is challenging in view of the multitude of intriguing possibilities, the background heterogeneity of leukemias, and the current absence of compelling preclinical models (Schein 2001).

\section{Clofarabine}

The nucleoside analog family includes the guanosine analogs, thioguanine and mercaptopurine, which were among the first agents to show activity against ALL (Hitchings et al 1950; Elion et al 1951; Skipper et al 1954). The clinical use of the deoxyadenosine analogs cladribine and fludarabine has been restricted due to dose-limiting neurotoxicity (Kantarjian et al 2007). Clofarabine (2-chloro-2'-fluoro2'deoxy-9-B-D-arabinofuranosyladenine), a next-generation deoxyadenosine analog (see Figure 1), was rationally designed to incorporate the best qualities and improve the therapeutic index of cladribine and fludarabine. The drug received accelerated approval by the FDA in December 2004 and EMEA approval in 2006 for the treatment of patients with childhood ALL who have received two or more prior therapeutic regimens.

Clofarabine retains the 2-halogenated aglycone of the deoxyadenoside analogs cladribine and fludarabine (Figure 1), which confers resistance to cellular degradation by adenosine deaminase (Parker et al 1991, 1999; Pui et al 2005). However, clofarabine also possesses a second halogen atom (fluorine) at the $2^{\prime}$ carbon in the arabino configuration, which inhibits cleavage of the glycosidic bond by bacterial purine nucleoside phosphorylase and stabilizes the compound in acidic environments, thus improving oral bioavailability and preventing the 


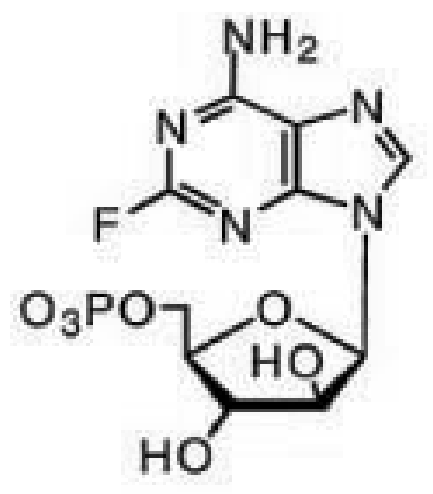

Fludarabine

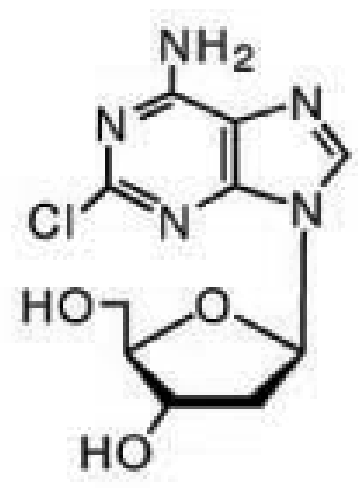

Cladribine

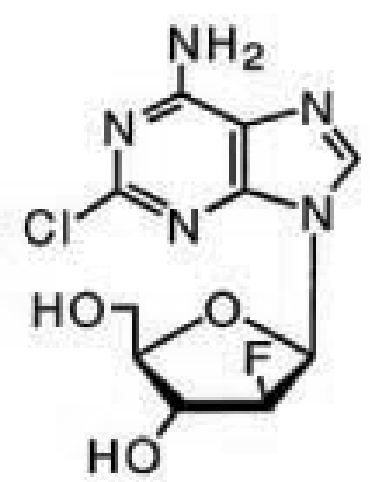

Clofarabine

Figure I Structures of clofarabine and other nucleoside analogs. Derived from Jeha et al (2004).

release of the neurotoxic halogenated adenine (Parker et al 1991; Carson et al 1992; Plunkett and Gandhi 2001; Jeha et al 2004; Jeha and Kantarjian 2007; Kantarjian et al 2007). In addition, clofarabine has greater affinity than cladribine or fludarabine for deoxycytidine kinase, the enzyme required for intracellular phosporylation to its monophosphorylated form. Clofarabine is then serially phosphorylated by other kinases to its active metabolite, clofarabine triphosphate (Bonate et al 2004, 2005). Clofarabine triphosphate is retained in acute leukemia cells for longer periods of time than the metabolites of the other purine nucleoside analogs (Xie and Plunkett 1995, 1996; Bonate et al 2006; Gandhi et al 2006,).

\section{Mechanism of action}

Purine analog triphosphates inhibit ribonucleotide reductase and are substrates for DNA polymerases for incorporation into DNA (Gandhi et al 2006). Fludarabine primarily inhibits DNA polymerases and cladribine mainly inhibits ribonucleotide reductase, while clofarabine inhibits both DNA polymerases and ribonucleotide reductase (Parker et al 1991, 1999; Xie and Plunkett 1995, 1996; Jeha et al 2004). Inhibition of DNA polymerases leads to impairment of DNA synthesis and/or repair through the incorporation of clofarabine monophosphate into the DNA chain (Xie and Plunkett 1996; Bonate et al 2006). Inhibition of ribonucleotide reductase depletes the deoxynucleotide pool primarily of dCTP and dATP (Xie and Plunkett 1996; Bonate et al 2006). The reduction in dCTP concentrations limits DNA synthesis, while the reduction in dATP creates a favorable environment for clofarabine triphosphate to compete with
dATP for incorporation into DNA (Xie and Plunkett 1996; Bonate et al 2006). A third mechanism of action is induction of apoptosis initiated by DNA strand breaks, resulting in the release of cytochrome $\mathrm{c}$ and activation of apoptotic pathways (Genini et al 2000; Bonate et al 2006). Clofarabine has also been shown to act directly on the mitochondria in primary chronic lymphocytic leukemia (CLL) cells, altering the transmembrane potential and releasing pro-apoptotic mitochondrial factors, which may explain the mechanism of cell death in cells not actively synthesizing DNA for cell division or DNA repair. This direct mitochondrial activity was not seen with fludarabine and may partly explain the enhanced cytotoxicity of clofarabine (Carson et al 1992; Genini et al 2000; Bonate et al 2006).

\section{Pharmacodynamics}

In the blast cells of adults with refractory leukemias, the accumulation of clofarabine triphosphate was associated with a decrease in DNA synthesis (Gandhi et al 2003). DNA synthesis was $75 \%$ to $95 \%$ inhibited at the end of an infusion with clofarabine at doses ranging from 22.5 to $55 \mathrm{mg} / \mathrm{m}^{2}$. At 24 hours, partial recovery of DNA synthesis occurred in the blasts of patients treated with 22.5 and $30 \mathrm{mg} / \mathrm{m}^{2}$, while the inhibition of DNA synthesis was maintained at 24 hours after $40-55 \mathrm{mg} / \mathrm{m}^{2}$ doses (Gandhi et al 2003; Kantarjian et al 2007).

\section{Pharmocokinetics and metabolism (Table 2)}

The population pharmacokinetics of plasma clofarabine and intracellular clofarabine triphosphate was studied in 
Table 2 Pharmacokinetics of clofarabine

\begin{tabular}{ll}
\hline Variable & Value \\
\hline Volume of distribution $\left(\mathrm{L} / \mathrm{m}^{2}\right)$ & 172 \\
Protein binding (\%) & 47 \\
Terminal half-life (hours) & 5.2 \\
Elimination & via urine $(49 \%-60 \%)$ \\
Systemic clearance $\left(\mathrm{L} / \mathrm{h} / \mathrm{m}^{2}\right)$ & 28.8 \\
Metabolism & Phosphorylated by deoxycytidine \\
& kinase phosphorylated to active \\
& metabolite clofarabine triphosphate \\
\hline
\end{tabular}

Derived from data of Bonate et al 2004.

40 pediatric patients aged $2-9$ years ( 21 males/19 females) with relapsed or refractory ALL or AML. Clofarabine pharmacokinetics was weight dependent, suggesting the importance of dosing on a body surface area (BSA) basis, although similar concentrations were obtained over a wide range of BSAs at the given $52 \mathrm{mg} / \mathrm{m}^{2}$ dose (Bonate et al 2004). Clofarabine was $47 \%$ bound to plasma proteins, mainly albumin. Systemic clearance and volume of distribution at steady state were estimated to be $28.8 \mathrm{~L} / \mathrm{h} / \mathrm{m}^{2}$ and $172 \mathrm{~L} / \mathrm{m}^{2}$, respectively. The terminal half-life was estimated to be 5.2 hours (Bonate et al 2004). The half-life of the active clofarabine triphosphate metabolite could not be adequately characterized in the pediatric phase 2 studies but was estimated to be greater than 24 hours (Bonate et al 2004). It is believed that the conversion from the monophosphate to the diphosphate moiety is the rate-limiting step in the formation of the triphosphate. It appears that clofarabine triphosphate formation is very rapid, and once formed, it remains trapped in the cell with a prolonged elimination half-life (Xie and Plunkett 1995; Bonate et al 2004). In pediatric patients $49 \%-60 \%$ of the clofarabine dose is excreted unchanged in the urine. The pathways of non-renal elimination remain unknown (Gandhi et al 2003; Bonate et al 2004). Clofarabine does not appear to be metabolized by the cytochrome $\mathrm{P} 450$ enzymes and CYP450 inhibitors and inducers are not likely to affect the metabolism of clofarabine, although no clinical drug-drug interaction studies have been conducted to date $\left(\right.$ Clolar $^{\circledR}$ package insert).

\section{Special populations}

Clofarabine pharmacokinetics is similar in patients with ALL and AML or in males and females. The pharmacokinetics of clofarabine remains to be evaluated in patients with renal or hepatic dysfunction (Bonate et al 2004). According to the manufacturer, its use in patients with hepatic or renal dysfunction should be undertaken only with the greatest caution.
Clofarabine was found to be teratogenic in reproductive studies conducted in rats and rabbits, with reduced fetal body weight, increased fetal loss, and skeletal malformations observed. Therefore, although there are no adequate studies in pregnant women using clofarabine, women of childbearing potential should be advised to avoid becoming pregnant while receiving treatment with clofarabine (Clolar ${ }^{\circledR}$ package insert).

\section{Efficacy studies}

\section{Phase I studies in pediatrics}

Based on a $16 \%$ overall response rate seen in adults with leukemia in a phase I single-agent trial of clofarabine (Gandhi et al 2003; Kantarjian et al 2003b; Faderl et al 2005a), a pediatric phase I trial was conducted to define the dose-limiting toxicity (DLT) and maxinimu tolerated dose (MTD) for children with acute leukemia (Jeha et al 2004). Six dose levels, ranging from 11.25 to $70 \mathrm{mg} / \mathrm{m}^{2}$ for 5 days, were studied in 25 pediatric patients (8 patients with AML and 17 patients with ALL). Most patients were heavily pretreated, with $36 \%$ having undergone prior stem cell transplantation (Jeha et al 2004). Hyperbilirubinemia and skin rash were dose-limiting and the recommended dose schedule for the phase 2 pediatric studies was $52 \mathrm{mg} / \mathrm{m}^{2}$ daily for 5 days. Complete response (CR) was reported in $4(24 \%)$ of 17 patients with ALL (Table 3). One patient $(6 \%)$ had a partial response (PR), for an overall response rate (CR + PR) for ALL of 30\%. Complete responses were seen at $30 \mathrm{mg} / \mathrm{m}^{2}, 40 \mathrm{mg} / \mathrm{m}^{2}$, and $52 \mathrm{mg} / \mathrm{m}^{2}$ (Jeha et al 2004). Responders included a 13-year-old female with $\mathrm{t}(9,22)$ refractory to 3 consecutive induction attempts including idarubicin, fludarabine, and cytarabine, as well as a 17-year-old female with T-cell ALL refractory to 3 consecutive inductions including nelarabine (Jeha et al 2004). The overall response rate for patients with AML was $38 \%$ (Table 3 ). One out of 8 patients (13\%) achieved CR and 2 patients (25\%) achieved PR (Jeha et al 2004).

\section{Phase II studies in children with acute leukemia}

A phase II, open-label, multicenter clofarabine study was conducted in 61 pediatric patients in the US with refractory

Table 3 Response to clofarabine in phase I trials

\begin{tabular}{llll}
\hline \multirow{2}{*}{ Response } & \multicolumn{3}{l}{ Responses/Total (\%) } \\
\cline { 2 - 4 } & ALL & AML & Total \\
\hline CR & $4 / 17(24 \%)$ & $1 / 8(13 \%)$ & $5 / 25(20 \%)$ \\
PR & $1 / 17(6 \%)$ & $2 / 8(25 \%)$ & $3 / 25(12 \%)$ \\
CR + PR & $5 / 17(30 \%)$ & $3 / 8(38 \%)$ & $8 / 25(32 \%)$ \\
\hline
\end{tabular}

Derived from data of Jeha et al (2004).

Abbreviations: $\mathrm{CR}$, complete response; $\mathrm{PR}$, partial response. 
or relapsed ALL at $52 \mathrm{mg} / \mathrm{m}^{2}$ for 5 days (Jeha et al 2006b). Patients were heavily pretreated, with a median of 3 prior induction regimens. Approximately one-third had received at least 1 prior transplantion, and $57 \%$ were refractory to the last therapeutic regimen. Twelve of 61 ALL patients achieved complete remission (CR) or complete remission without platelet recovery (CRp). Only 1 of 42 AML patients achieved CRp. Of those who were refractory to their most recent prior regimen, the overall response rate was $17 \%$. There were an additional $10 \%$ of patients who achieved a partial response (PR). The median duration of response for patients with ALL who achieved at least a partial response was 9.7 weeks. Responses were observed in patients with both B-and T-lineage ALL, and in patients with various cytogenetic abnormalities. Nine of the 61 ALL patients proceeded to HSCT, with no apparent increase in transplantation-related toxicity. The median duration of CR was 7 months in patients receiving transplants (Jeha et al 2006b; Jeha and Kantarjian 2007). The most common adverse events, grade 3 or higher, were fever with neutropenia, anorexia, hypotension, and nausea. Similar response rates were seen in a European phase II trial in pediatric patients with ALL, with a reported CR + CRp rate of $28 \%$ (Kearns et al 2006).

Forty-two children with AML were reported in a separate pediatric phase II clofarabine trial. The overall response rate in the heavily pretreated pediatrics was $26 \%$ ( $1 \mathrm{CRp}$ and 8 PRs) (Table 4). The median duration of remission was 16.2 weeks and reported toxicities were similar to patients with ALL (Jeha et al 2006b).

\section{Combination studies}

The pediatric phase I and phase II single agent trials determined that clofarabine was active in pediatric patient with relapsed and refractory ALL with an acceptable toxicity profile. Rational combinations of clofarabine with other agents are being investigated in order to optimize clinical

Table 4 Response to clofarabine in phase II trials in United States

\begin{tabular}{lll}
\hline & \multicolumn{2}{l}{ Responses/Total (\%) } \\
\cline { 2 - 3 } Response & ALL & AML \\
\hline CR & $7 / 6 I(I 2 \%)$ & \\
CRp & $5 / 6 I(8 \%)$ & $1 / 35(3 \%)$ \\
PR & $6 / 6 I(10 \%)$ & $8 / 35(23 \%)$ \\
CR + CRp & $12 / 6 I(20 \%)$ & $1 / 35(3 \%)$ \\
CR + CRp + PR & $18 / 6 I(30 \%)$ & $9 / 35(26 \%)$ \\
\hline
\end{tabular}

Derived from data of Jeha et al (2006 and 2004).

Abbreviations: $\mathrm{CR}$, complete remission; $\mathrm{CRp}$, complete remission without full platelet recovery; PR, partial remission. benefit. In vitro data suggest that clofarabine, an inhibitor of DNA repair, may enhance the cytotoxicity of DNA-damaging agents such as cyclophosphamide (Yamauchi et al 2001). The combination of cyclophosphamide and etoposide is utilized during consolidation therapy for pediatric ALL patients with ALL in first relapse (Children's Oncology Group Protocol AALL01P2), and a similar ifosfamide/etoposide combination is active in multiply relapsed ALL (Crooks and Sato 1995). A pilot phase I study of clofarabine used in combination with cyclophosphamide and etoposide is ongoing to determine the MTD and the dose-limiting toxicities of the combination. Once etoposide and cyclophosphamide were escalated to their target doses $\left(100 \mathrm{mg} / \mathrm{m}^{2} /\right.$ day and $440 \mathrm{mg} /$ $\mathrm{m}^{2} /$ day, respectively in cohort 3 ), clofarabine was increased to $30 \mathrm{mg} / \mathrm{m}^{2} /$ day in cohort 4 and would be increased to $40 \mathrm{mg} / \mathrm{m}^{2}$ in cohort 5 (Hijiya et al 2007). To date, 19 patients (15 ALL, 4 AML) have been enrolled in the first 4 dose cohorts (Table 5). Seventeen patients (14 ALL, 3 AML) have response data available. The median number of prior regimens was 2. Seven of 14 evaluable ALL patients achieved a CR and 2 ALL patients achieved a CRp, for an overall response rate of $64 \%$ among pediatric patients with ALL. Three out of 3 patients with AML achieved a CRp. One patient in cohort 4 experienced a grade 3 elevation of lipase and possible veno-occlusive disease. Febrile neutropenia was a common toxicity. Phase 2 studies of this combination will take place once the MTD is determined (Hijiya et al 2007).

Other studies are investigating whether clofarabine, a potent inhibitor of ribonucleotide reductase, will modulate intracellular cytarabine (Ara-c) triphosphate accumulation and increase the cytotoxicity of Ara-c (Cooper et al 2005; Faderl et al 2005b, 2006). A phase 1-2 adult study in 32 adults with relapsed and refractory acute leukemias demonstrated that the combination of clofarabine at the adult MTD dose level of $40 \mathrm{mg} / \mathrm{m}^{2} /$ day for 5 days (days 2-6) followed 4 hours later by cytarabine $1 \mathrm{~g} / \mathrm{m}^{2}$ administered as a 2-hour intravenous infusion for 5 days (days 1-5) was safe and effective (Faderl et al 2005b). The overall response rate was $38 \%$ with 1 death during induction. Adverse events were mainly less than or equal to grade 2, including transient liver enzyme elevations, nausea, diarrhea, skin rash, mucositis, and palmo-plantar erythrodysesthesias. A Children's Oncology Group (COG) study (AAML0523) is underway to explore the safety and effecacy of this combination.

\section{Safety and tolerability}

Clofarabine is tolerated at 20 times higher doses in leukemia patients than in patients with solid tumors, where 
Table 5 Response to combination of clofarabine/cyclophosphamide/ etoposide in pediatric leukemia patients

\begin{tabular}{lll}
\hline & \multicolumn{2}{l}{ Responses/Total (\%) } \\
\cline { 2 - 3 } Response & ALL & AML \\
\hline CR & $7 / 14(50 \%)$ & $3 / 3(100 \%)$ \\
CRp & $2 / 14(8 \%)$ & \\
PR & & $3 / 3(100 \%)$ \\
CR + CRp & $9 / 14(64 \%)$ & \\
\hline
\end{tabular}

Adapted from Hijaya et al 2007.

Abbreviations: $C R$, complete remission; $C R p$, complete remission without full platelet recovery; PR, partial remission.

myelosuppression is dose-limiting (Kantarjian et al 2003b). The dose-limiting toxicity in pediatric leukemia patients is hyperbilirubinemia and transient elevations in liver enzymes, which reversed by day 14, as well as grade 3 skin rash (Jeha et al 2004). Some patients experienced CNS irritability with the 1-hour infusion, which resolved when the clofarabine was given over 2 hours. Other adverse events greater than or equal to grade 3 which occurred in $10 \%$ or more of patients included febrile neutropenia, anorexia, hypotension, nausea, sepsis, and pleural effusion (Jeha et al 2006a, b). Drug-related fevers and skin rash generally responded to antihistamines and may require low-dose corticosteroids, once infection is excluded. Clofarabine administration has been associated with rapid decreases in blasts even in non-responders; this may be accompanied by tumor lysis syndrome or systemic inflammatory response and capillary leak syndrome, with respiratory distress, fever, and hypotension. Cytokine-release like events may have contributed to a decline in cardiac function that was observed in several patients.

\section{Drug interactions and concomitant medications}

No clinical drug-drug interaction studies have been conducted to date. Clofarabine is primarily excreted by the kidneys, thus it may potentiate the toxicity of known nephrotoxic drugs, including aminoglycoside antibiotics. Tumor lysis and hyperuricemia may also contribute to renal toxicity. The concomitant use of medications known to induce hepatic toxicity should also be avoided, since hepato-biliary toxicities were observed in pediatric trials. In addition, patients taking medications known to affect blood pressure or cardiac function should be closely monitored during the administration of clofarabine, as symptoms of cytokine release and capillary leak syndrome, including hypotension, tachycardia, and pulmonary edema, have been reported in patients undergoing treatment with clofarabine (Clolar ${ }^{\circledR}$ package insert).

\section{Dosage}

The recommended dose and schedule of clofarabine as a single agent for pediatric patients is $52 \mathrm{mg} / \mathrm{m}^{2}$ administered by intravenous (IV) infusion over 2 hours daily for 5 consecutive days. The dosage is based on the patient's BSA, calculated using the actual height and weight before the start of each cycle. Treatment cycles are repeated following blood count recovery or return to baseline organ function, approximately every $2-6$ weeks (Clolar ${ }^{\circledR}$ package insert). To prevent drug incompatibilities, no other medications should be administered through the same intravenous line. Clofarabine should be administered along with IV infusion fluids to reduce the effects of tumor lysis and other adverse events. Allopurinol may be administered prophylactically if hyperuricemia is expected. The use of prophylactic steroids (eg, $100 \mathrm{mg} / \mathrm{m}^{2}$ hydrocortisone on days 1 through 3) may be of benefit in preventing signs or symptoms of systemic inflammatory response syndrome (SIRS)/capillary leak syndrome. If signs or symptoms of SIRS or capillary leak occur (eg, hypotension), clofarabine should be discontinued and the use of steroids, diuretics, and albumin considered. According to the manufacturer, clofarabine can be re-instituted when the patient is stable and organ function has returned to baseline, generally with a $25 \%$ dose reduction.

\section{Conclusions}

Better use of existing drugs has dramatically improved survival rates for childhood acute leukemias over the past few decades. However, relapsed and refractory childhood leukemias remain a significant therapeutic challenge. Relapse after multi-agent therapy may arise from mutations in shared cell-death pathways, rather than alterations in specific drug activation, drug elimination, or drug targets. In addition to resistant disease, heavily pretreated patients often have underlying infection and pre-existing organ dysfunction. New candidate agents need be evaluated in combination. Agents may have substantial single agent activity, yet contribute nothing in combination, eg, ifosfamide in rhabdomyosarcoma (Miser et al 1987; Crist et al 2001). Conversely, an agent may have no single agent activity, yet still contribute in combination, eg, leukovorin in colon cancer (Iqbal and Lenz 2004).

Clofarabine is safe and active in both myeloid and lymphoid relapsed/refractory pediatric acute leukemias in single agent phase I and II trials. Myelosuppression was an acceptable effect in this population, and the neurotoxicity associated with previous generation nucleoside analogs was not observed with clofarabine. Responses in relapsed 
pediatric ALL were durable and allowed some patients to successfully proceed to transplantation. Clofarabine has been approved by the FDA for the treatment of pediatric patients with relapsed or refractory ALL after at least 2 prior therapeutic regimens based on the induction of complete responses. Over the past decade, clofarabine has been the only anti-cancer agent to receive primary indication for use in children.

Rational combinations of clofarabine with other active agents in refractory leukemias are currently under investigation. The early results of the phase I study of clofarabine in combination with cyclophosphamide and etoposide in pediatric patients with relapsed or refractory ALL or AML indicate that this combination shows significant activity in children with both subtypes of refractory leukemia and is well tolerated (Hijiya et al 2007). Pediatric studies of clofarabine in combination with cytarabine to enhance intracellular Ara-C triphosphate accumulation are also in progress. Such rationally designed combinations of clofarabine with standard agents may have a role in frontline therapy for newly diagnosed patients with higher risk disease. As of September, 2007 COG plans to study clofarabine-based combinations in a randomized phase III trial in patients with "very high risk" B-precursor ALL, ie, hypodiploidy, higher end-induction MRD, and/or induction failure. The proposed study tests a clofarabine blocks against the cyclophophamide/ara-c/thiopurine blocks that occupy 3 of the first 6 months of post-induction intensification. Plans in AML await completion of the current COG trials, AAML0523.

\section{Disclosures}

TMH has no conflicts of interest. PSG is on the speakers' bureaus for Genzyme, Sanofi-Aventis, and Enzon.

\section{References}

Aladjidi N, Auvrignon A, Leblanc T, et al. 2003. Outcome in children with relapsed acute myeloid leukemia after initial treatment with the French Leucemie Aique Myeloide Enfant (LAME) 89/91 protocol of the French Society of Pediatric Hematology and Immunology. J Clin Oncol, 21:4377-85.

Arceci RJ, Sande J, Lange B, et al. 2005. Safety and efficacy of gemtuzumab ozogamicin in pediatric patients with advanced CD33+ acute myeloid leukemia. Blood, 106:1183-8.

Bader P, Hancock J, Kreyenberg H, et al. 2002. Minimal residual disease (MRD) status prior to allogeneic stem cell transplantation is a powerful predictor for post-transplant outcome in children with ALL. Leukemia, 16:1668-72.

Bonate PL, Arthaud L, Cantrell WR Jr, et al. 2006. Discovery and development of clofarabine:a nucleoside analogue for treating cancer. Nat Rev Drug Discov, 5:855-63.

Bonate PL, Arthaud L, Stuhler J, et al. 2005. The distribution, metabolism, and elimination of clofarabine in rats. Drug Metab Dispos, 33:739-48.
Bonate PL, Craig A, Gaynon P, et al. 2004. Population pharmacokinetics of clofarabine, a second-generation nucleoside analog, in pediatric patients with acute leukemia. J Clin Pharmacol, 44:1309-22.

Burnett AK, Kell WJ, Goldstone AH, et al. 2006. The Addition of Gemtuzumab Ozogamicin to Induction Chemotherapy for AML Improves Disease Free Survival without Extra Toxicity: Preliminary Analysis of 1115 Patients in the MRC AML15 Trial. ASH Annual Meeting Abstracts, 108:13.

Carson DA, Wasson DB, Esparza LM, et al. 1992. Oral antilymphocyte activity and induction of apoptosis by 2-chloro-2'arabino-fluoro-2'-deoxyadenosine. Proc Natl Acad Sci, USA, 89:2970-4

Castellino SM, Alonzo TA, Buxton A, et al. 2007. Outcomes in childhood AML in the absence of transplantation in first remission-Children's Cancer Group (CCG) studies 2891 and CCG 213. Pediatr Blood Cancer, Jan 24 [epub ahead of print].

Chessells JM. 1998. Relapsed lymphoblastic leukaemia in children:a continuing challenge. Br J Haematol, 102:423-38.

Chessells JM, Veys P, Kempski H, et al. 2003. Long-term follow-up of relapsed childhood acute lymphoblastic leukaemia. Br J Haematol, 123:396-405.

Clolar $^{\circledR}$ package insert.

Cooper T, Ayres M, Nowak B, et al. 2005. Biochemical modulation of cytarabine triphosphate by clofarabine. Cancer Chemother Pharmacol, 55:361-8.

Coustan-Smith E, Gajjar A, Hijiya N, et al. 2004. Clinical significance of minimal residual disease in childhood acute lymphoblastic leukemia after first relapse. Leukemia, 18:499-504.

Creutzig U, Zimmermann M, Lehrnbecher T, et al. 2006. Less toxicity by optimizing chemotherapy, but not by addition of granulocyte colony-stimulating factor in children and adolescents with acute myeloid leukemia:results of AML-BFM 98. J Clin Oncol, 24:4499-506

Creutzig U, Zimmermann M, Ritter J, et al. 2005. Treatment strategies and long-term results in paediatric patients treated in four consecutive AML-BFM trials. Leukemia, 19:2030-42.

Crist WM, Anderson JR, Meza JL, et al. 2001. Intergroup rhabdomyosarcoma study-IV:results for patients with nonmetastatic disease. $J$ Clin Oncol, 19:3091-102.

Crooks GM, Sato JK. 1995. Ifosfamide and etoposide in recurrent childhood acute lymphoblastic leukemia. J Pediatr Hematol Oncol, 17:34-8.

Eapen M, Raetz E, Zhang MJ, et al. 2006. Outcomes after HLAmatched sibling transplantation or chemotherapy in children with B-precursor acute lymphoblastic leukemia in a second remission: a collaborative study of the Children's Oncology Group and the Center for International Blood and Marrow Transplant Research. Blood, 107:4961-7.

Eckert C, Biondi A, Seeger K, et al. 2001. Prognostic value of minimal residual disease in relapsed childhood acute lymphoblastic leukaemia. Lancet, 358:1239-41.

Einsiedel HG, von Stackelberg A, Hartmann R, et al. 2005. Long-term outcome in children with relapsed ALL by risk-stratified salvage therapy: results of trial acute lymphoblastic leukemia-relapse study of the Berlin-Frankfurt-Munster Group 87. J Clin Oncol, $23: 7942-50$.

Elion GB, Hitchings GH, Vanderwerff H. 1951. Antagonists of nucleic acid derivatives. VI. Purines. J Biol Chem, 192:505-18.

Faderl S, Gandhi V, Keating MJ, et al. 2005a. The role of clofarabine in hematologic and solid malignancies - development of a next-generation nucleoside analog. Cancer, 103:1985-95.

Faderl S, Gandhi V, O'Brien S, et al. 2005b. Results of a phase 1-2 study of clofarabine in combination with cytarabine (ara-C) in relapsed and refractory acute leukemias. Blood, 105:940-7.

Faderl S, Verstovsek S, Cortes J, et al. 2006. Clofarabine and cytarabine combination as induction therapy for acute myeloid leukemia (AML) in patients 50 years of age or older. Blood, 108:45-51. 
Gandhi V, Kantarjian H, Faderl S, et al. 2003. Pharmacokinetics and pharmacodynamics of plasma clofarabine and cellular clofarabine triphosphate in patients with acute leukemias. Clin Cancer Res, 9:6335-42.

Gandhi V, Plunkett W, Bonate PL, et al. 2006. Clinical and pharmacokinetic study of clofarabine in chronic lymphocytic leukemia:strategy for treatment. Clin Cancer Res, 12:4011-17.

Gaynon PS. 2005. Childhood acute lymphoblastic leukaemia and relapse. Br J Haematol, 131:579-87.

Gaynon PS, Harris RE, Altman AJ, et al. 2006. Bone marrow transplantation versus prolonged intensive chemotherapy for children with acute lymphoblastic leukemia and an initial bone marrow relapse within 12 months of the completion of primary therapy:Children's Oncology Group study CCG-1941. J Clin Oncol, 24:3150-6.

Gaynon PS, Qu RP, Chappell RJ, et al. 1998. Survival after relapse in childhood acute lymphoblastic leukemia:impact of site and time to first relapse - the Children's Cancer Group Experience. Cancer, 82:1387-95.

Genini D, Adachi S, Chao Q, et al. 2000. Deoxyadenosine analogs induce programmed cell death in chronic lymphocytic leukemia cells by damaging the DNA and by directly affecting the mitochondria. Blood, 96:3537-43.

Gibson BE, Wheatley K, Hann IM, et al. 2005. Treatment strategy and long-term results in paediatric patients treated in consecutive UK AML trials. Leukemia, 19:2130-8.

Goulden N, Bader P, Van Der Velden V, et al. 2003. Minimal residual disease prior to stem cell transplant for childhood acute lymphoblastic leukaemia. Br J Haematol, 122:24-9.

Grovas A, Fremgen A, Rauck A, et al. 1997. The National Cancer Data Base report on patterns of childhood cancers in the United States. Cancer, 80:2321-32.

Hijiya N, Franklin J, Rytting M, et al. 2007. A phase I study of clofarabine in combination with cyclophosphamide and etoposide:a new regimen in pediatric patients with relapsed or refractory acute leukemia. Proc Am Soc Clin Oncol, 26:abstract \#9529.

Hitchings GH, Elion GB, Falco EA, et al. 1950. Studies on analogs of purines and pyrimidines. Ann N Y Acad Sci, 52:1318-35.

Iqbal S, Lenz HJ. 2004. Integration of novel agents in the treatment of colorectal cancer. Cancer Chemother Pharmacol, 54(Suppl 1): S32-39.

Jeha S, Gandhi V, Chan KW, et al. 2004. Clofarabine, a novel nucleoside analog, is active in pediatric patients with advanced leukemia. Blood, 103:784-9.

Jeha S, Gaynon PS, Razzouk BI, et al. 2006a. Phase II study of clofarabine in pediatric patients with refractory or relapsed acute lymphoblastic leukemia. J Clin Oncol, 24:1917-23.

Jeha S, Kantarjian H. 2007. Clofarabine for the treatment of acute lymphoblastic leukemia. Expert Rev Anticancer Ther, 7:113-118.

Jeha S, Razzouk B, Gaynon P, et al. 2006b. Clofarabine in children with refractory/relapsed acute leukemia: results of 2 phase ii open label studies. ASH Annual Meeting Abstracts, 108:4460.

Jemal A, Tiwari RC, Murray T, et al. 2004. Cancer statistics, 2004. CA Cancer J Clin, 54:8-29.

Kantarjian H, Gandhi V, Cortes J, et al. 2003a. Phase 2 clinical and pharmacologic study of clofarabine in patients with refractory or relapsed acute leukemia. Blood, 102:2379-86.

Kantarjian HM, Gandhi V, Kozuch P, et al. 2003b. Phase I clinical and pharmacology study of clofarabine in patients with solid and hematologic cancers. J Clin Oncol, 21:1167-73.

Kantarjian H, Jeha S, Gandhi V, et al. 2007. Clofarabine:Past, present, and future. Leuk Lymphoma, Aug 13:1-9 [epub ahead of print].

Kearns P, Michel G, Nelken B, et al. 2006. BIOV-111a European phase II trial of clofarabine (Evoltra) in refractroy and relapsed childhood acute lymphoblastic leukemia. Blood, 108:527a (abstract \#1859).

Knechtli CJ, Goulden NJ, Hancock JP, et al. 1998. Minimal residual disease status before allogeneic bone marrow transplantation is an important determinant of successful outcome for children and adolescents with acute lymphoblastic leukemia. Blood, 92:4072-9.
Kurtzberg J. 2007. The long and winding road of the clinical development of Nelarabine. Leuk Lymphoma, 48:1-2.

Levis M, Smith BD, Beran M, et al. 2005. A randomized, open-label study of lestaurtinib (CEP-701), an oral FLT3 inhibitor, administered in sequence with chemotherapy in patients with relapsed AML harboring FLT3 activating mutations: clinical response correlates with successful FLT3 inhibition. ASH Annual Meeting Abstracts, 106:403.

Miser JS, Kinsella TJ, Triche TJ, et al. 1987. Ifosfamide with mesna uroprotection and etoposide:an effective regimen in the treatment of recurrent sarcomas and other tumors of children and young adults. J Clin Oncol, 5:1191-8.

Nguyen K, Cheng S-C, Raetz E, et al. 2006. Factors influencing survival after relapse from childhood ALL: A Children's Oncology Group study. ASH Annual Meeting Abstracts, 108:1855.

Parker WB, Shaddix SC, Chang CH, et al. 1991. Effects of 2-chloro-9(2-deoxy-2-fluoro-beta-D-arabinofuranosyl)adenine on K562 cellular metabolism and the inhibition of human ribonucleotide reductase and DNA polymerases by its 5'-triphosphate. Cancer Res, 51:2386-94.

Parker WB, Shaddix SC, Rose LM, et al. 1999. Comparison of the mechanism of cytotoxicity of 2-chloro-9-(2-deoxy-2- fluoro-beta-D-arabinofuranosyl) adenine, 2-chloro-9-(2-deoxy-2-fluoro- beta-D-ribofuranosyl)adenine, and 2-chloro-9-(2-deoxy-2,2-difluoro- beta-D-ribofuranosyl)adenine in CEM cells. Mol Pharmacol, 55:515-20.

Plunkett W, Gandhi V. 2001. Purine and pyrimidine nucleoside analogs. Cancer Chemother Biol Response Modif, 19:21-45.

Pollard JA, Alonzo TA, Gerbing RB, et al. 2006. FLT3 internal tandem duplication in $\mathrm{CD} 34+/ \mathrm{CD} 33$ - precursors predicts poor outcome in acute myeloid leukemia. Blood, 108:2764-9.

Powell BL, Moser B, Stock W, et al. 2006. Preliminary results from the North American Acute Promyelocytic Leukemia (APL) study C9710. ASH Annual Meeting Abstracts, 108:566.

Powell B, Moser B, Stock W, et al. 2007. Effect of consolidation with arsenic trioxide (As 2 O 3) on event-free survival (EFS) and overall survival (OS) among patients with newly diagnosed acute promyelocytic leukemia (APL):North American Intergroup Protocol C9710. Proc Am Soc Clin Oncol, 26:2.

Pui, C.H, Jeha, S. 2005b. Clofarabine. Nat Rev Drug Discov, (Suppl): S12-13.

Pui CH, Jeha S, Kirkpatrick P. 2005a. Clofarabine. Nat Rev Drug Discov, 4:369-70.

Raetz EA, Borowitz MJ, Devidas M, et al. 2006. Outcomes of children with first marrow relapse: Results from Children's Oncology Group (COG) study AALL01P2. ASH Annual Meeting Abstracts, 108:1871.

Reinhardt D, Diekamp S, Fleischhack G, et al. 2004. Gemtuzumab ozogamicin (Mylotarg) in children with refractory or relapsed acute myeloid leukemia. Onkologie, 27:269-72.

Schein PS. 2001. The case for a new national program for the development of cancer therapeutics. J Clin Oncol, 19:3142-53.

Schultz KR, Pullen DJ, Sather HN, et al. 2007. Risk- and response-based classification of childhood B-precursor acute lymphoblastic leukemia:a combined analysis of prognostic markers from the Pediatric Oncology Group (POG) and Children's Cancer Group (CCG). Blood, 109:926-35.

Sievers EL, Larson RA, Stadtmauer EA, et al. 2001. Efficacy and safety of gemtuzumab ozogamicin in patients with CD33-positive acute myeloid leukemia in first relapse. J Clin Oncol, 19:3244-54.

Skipper HE, Thomson JR, Elion GB. et al. 1954. Observations on the anticancer activity of 6-mercaptopurine. Cancer Res, 14:294-8.

Tallman MS. 2006. New agents for the treatment of acute myeloid leukemia. Best Pract Res Clin Haematol, 19:311-20.

Webb DK. 1999. Management of relapsed acute myeloid leukaemia. $\mathrm{Br} J$ Haematol, 106:851-9.

Wells RJ, Adams MT, Alonzo TA, et al. 2003. Mitoxantrone and cytarabine induction, high-dose cytarabine, and etoposide intensification for pediatric patients with relapsed or refractory acute myeloid leukemia: Children's Cancer Group Study 2951. J Clin Oncol, 21:2940-7.

Xie C, Plunkett W. 1995. Metabolism and actions of 2-chloro-9-(2-deoxy2-fluoro-beta-D- arabinofuranosyl)-adenine in human lymphoblastoid cells. Cancer Res, 55:2847-52. 
Xie KC, Plunkett W. 1996. Deoxynucleotide pool depletion and sustained inhibition of ribonucleotide reductase and DNA synthesis after treatment of human lymphoblastoid cells with 2-chloro-9-(2-deoxy-2-fluorobeta-D-arabinofuranosyl) adenine. Cancer Res, 56:3030-7.

Yamauchi T, Nowak BJ, Keating MJ, et al. 2001. DNA repair initiated in chronic lymphocytic leukemia lymphocytes by 4-hydroperoxycyclophosphamide is inhibited by fludarabine and clofarabine. Clin Cancer Res, 7:3580-9.

Zwaan CM, Reinhardt D, Corbacioglu S, et al. 2003. Gemtuzumab ozogamicin: first clinical experiences in children with relapsed/refractory acute myeloid leukemia treated on compassionate-use basis. Blood, 101:3868-71. 